\title{
1 Homogeneous multifocal excitation for high-throughput super-resolution imaging
}

2 Dora Mahecic ${ }^{1,2 *}$, Davide Gambarotto ${ }^{3}$, Kyle M. Douglass ${ }^{1}$, Denis Fortun ${ }^{4}$, Niccoló

3 Banterle ${ }^{2,5}$, Maeva Le Guennec ${ }^{3}$, Khalid Ibrahim, Pierre Gönczy ${ }^{2,5}$, Virginie Hamel ${ }^{3}$, Paul

4 Guichard $^{3}$, Suliana Manley ${ }^{1,2 *}$

5

6

$7 \quad$ Affiliations

$8{ }^{1}$ Laboratory for Experimental Biophysics, Institute of Physics, Swiss Federal Institute of

9 Technology Lausanne (EPFL), Lausanne, Switzerland.

102 Swiss National Centre for Competence in Research (NCCR) in Chemical Biology,

11 University of Geneva, Geneva, Switzerland.

$12{ }^{3}$ Department of Cell Biology, Sciences III, University of Geneva, Geneva, Switzerland.

$13{ }^{4}$ ICube, CNRS, University of Strasbourg, Illkirch, France.

$14 \quad{ }^{5}$ Swiss Institute for Experimental Cancer Research, School of Life Sciences, Swiss Federal

15 Institute of Technology Lausanne (EPFL), Lausanne, Switzerland.

16

$17 \quad{ }^{*}$ Corresponding authors: suliana.manley@epfl.ch; dora.mahecic@epfl.ch

18 


\section{Abstract}

20 Super-resolution microscopies, which allow features below the diffraction limit to be

21 resolved, have become an established tool in biological research. However, imaging

22 throughput remains a major bottleneck in using them for quantitative biology, which requires

23 large datasets to overcome the noise of the imaging itself and to capture the variability

24 inherent to biological processes. Here, we develop a multi-focal flat illumination for field

25 independent imaging (mfFIFI) module, and integrate it into an instant structured illumination

26 microscope (iSIM). Our instrument extends the field of view (FOV) to $>100 \times 100 \mu m^{2}$ without

27 compromising image quality, and maintains high-speed $(100 \mathrm{~Hz})$, multi-color, volumetric

28 imaging at double the diffraction-limited resolution. We further extend the effective FOV by

29 stitching multiple adjacent images together to perform fast live-cell super-resolution imaging

30 of dozens of cells. Finally, we combine our flat-fielded iSIM setup with ultrastructure expansion microscopy (U-ExM) to collect 3D images of hundreds of centrioles in human cells, as well as of thousands of purified Chlamydomonas reinhardtii centrioles per hour at an effective resolution of $\sim 35 \mathrm{~nm}$. We apply classification and particle averaging to these

34 large datasets, allowing us to map the 3D organization of post-translational modifications of centriolar microtubules, revealing differences in their coverage and positioning.

Introduction

Super-resolution fluorescence techniques have enabled optical imaging beyond the diffraction limit. Two main illumination strategies are used to achieve super-resolution: wide-

39 field illumination is typically used for single-molecule localization microscopies (SMLM) 40 such as STORM ${ }^{1}$ and PALM $^{2}$, while patterned illumination is typically used for methods 41 such as structured illumination microscopy $(\mathrm{SIM})^{3,4}$ and stimulated emission depletion 42 (STED $)^{5}$. Initial implementations of these methods were relatively slow, in part due to the 43 use of the time domain to separate single molecules (SMLM), the need to collect multiple 44 images to cover Fourier space (SIM), or the scanning of a reduced excitation volume 
45 (STED). In both wide-field and patterned illumination, the imaging throughput - defined as

46 the area imaged per time - can be increased by parallelizing the acquisition, as long as the

47 necessary illumination can be maintained over a larger surface in the sample plane.

48 Extending the array of patterned excitation has been used to increase the speed or FOV of 49 confocal $^{6}$, STED $^{7,8}$ and SIM imaging ${ }^{9}$, but ensuring the quality of the extended pattern 50 across a larger FOV remains a limiting factor. For SMLM, flat-fielding approaches made it 51 possible to extend super-resolution imaging over $\sim 100 \times 100 \mu \mathrm{m}^{2} \mathrm{FOVs}^{10,11}$, but their transfer 52 to patterned illumination remains limited.

An entirely different approach to effectively achieve super-resolution modifies sample preparation rather than image acquisition. By physically increasing the size of the specimen in an isotropic manner via expansion microscopy ${ }^{12}$ (ExM), overall imaging throughput can be increased by opting for faster diffraction-limited microscopes ${ }^{12-14}$. Alternatively, combining ExM with existing super-resolution microscopies allows even 58 further improvement in resolution ${ }^{14-18}$, since the resolvable scale is reduced by the 59 expansion factor. However, expansion exacerbates limitations in imaging throughput since 60 the effective FOV size is divided by the expansion factor along each dimension ${ }^{19}$. 61 Therefore, fast, parallelized, super-resolution techniques with large FOVs present an advantage for imaging ExM samples, among other applications. The instant structured illumination microscope (iSIM) ${ }^{9}$ uses parallelization in the 64 form of multifocal excitation and optical image processing to achieve a two-fold 65 improvement in resolution, at frame rates reaching $100 \mathrm{~Hz}$. While this gives the iSIM good 66 potential for high-throughput super-resolution imaging, spatially varying illumination 67 produces a spatially dependent signal to noise ratio while restricting the imaging FOV to $\sim 50 \times 50 \mu \mathrm{m}^{2}$. Although solutions for producing homogeneous multi-focal excitation exist using diffractive optical elements ${ }^{20}$, spatial light modulators $(S L M)^{21}$, multi-mode fibers ${ }^{22}$ or

70 beam splitters ${ }^{23}$, each suffers from some combination of a limited number of excitation 71 spots $^{20,21,23}$, heterogeneity ${ }^{21,23}$ or low transmission efficiency ${ }^{20}$. 
In this work, we develop a flat-fielding element for multi-focal excitation, dubbed

$73 \mathrm{mfFIFI}$. Our design is based on the Koehler integrator $24-26$ and ensures that the

74 homogeneity, pitch and size of the excitation spots are optimized. We integrate mfFIFI into

75 an iSIM to engineer an instrument capable of 3D multi-color imaging with uniform quality

76 and doubled resolution over a $100 \times 100 \mu \mathrm{m}^{2}$ FOV. We further parallelize the acquisition

77 across multiple FOVs, stitching them together to create seamless montages of tens of

78 super-resolved living cells within seconds, enabled by the homogeneous illumination.

79 Finally, we combine the mfFIFI iSIM with ultrastructure expansion microscopy (U-ExM) ${ }^{31}$ to

80 perform high-throughput super-resolution imaging with an effective resolution of $\sim 35 \mathrm{~nm}$.

81 We use this to collect 3D images of hundreds of expanded centrioles in human cells, and

82 thousands of expanded purified Chlamydomonas reinhardtii centrioles. These particles are

83 averaged to reconstruct maps of post-translational modifications (PTMs) of centriolar

84 tubulin, revealing differences in the spatial organization of acetylation, monoglutamylation

85 and polyglutamylation.

\section{Results}

87 Design and implementation of homogeneous multifocal excitation

Generating a uniform irradiance over a large FOV while retaining the highest achievable resolution requires the following properties of the excitation focal spots in a multi-focal microscope: 1) homogeneous intensity, 2) constant pitch and 3) diffraction-

91 limited size across the field of view. Existing methods for generating uniform excitation are

92 often limited to producing a low number of excitation spots, with residual heterogeneity and

93 low transmission efficiency. Thus, we set out to extend the Koehler integrator ${ }^{24-26}$, which

94 averages over the spatial and angular distributions of the light source to homogenize the 95 beam, for use with multifocal excitation. 
a)

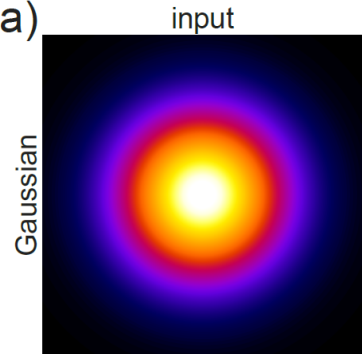

b)

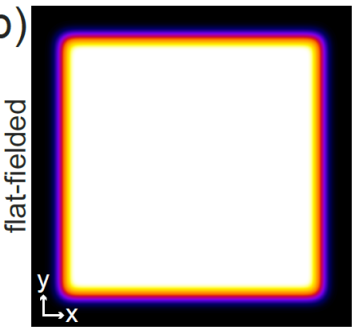

C)

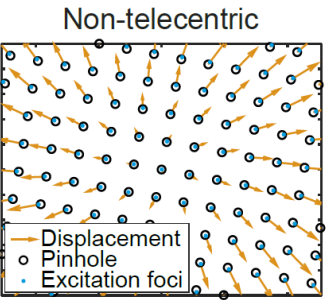

f)

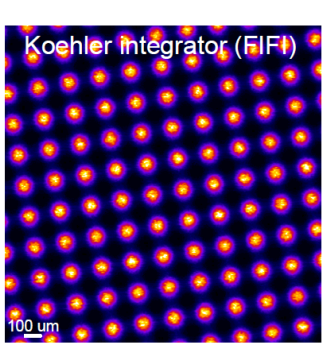

propagation
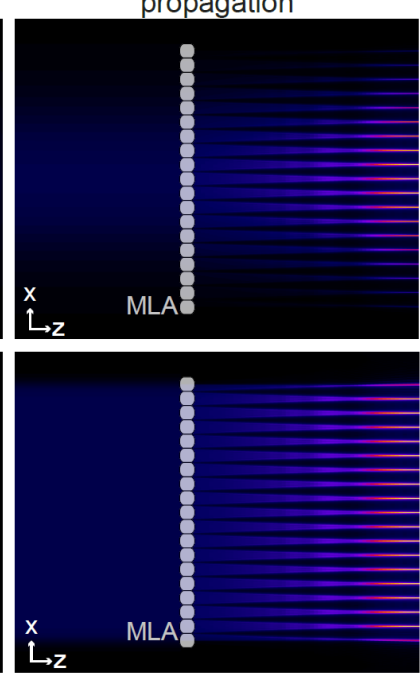

d) Telecentric
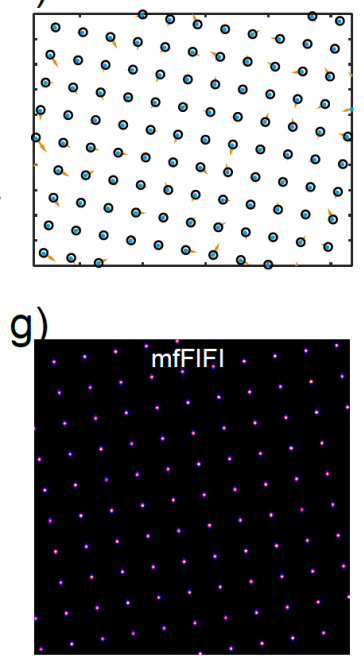

e)
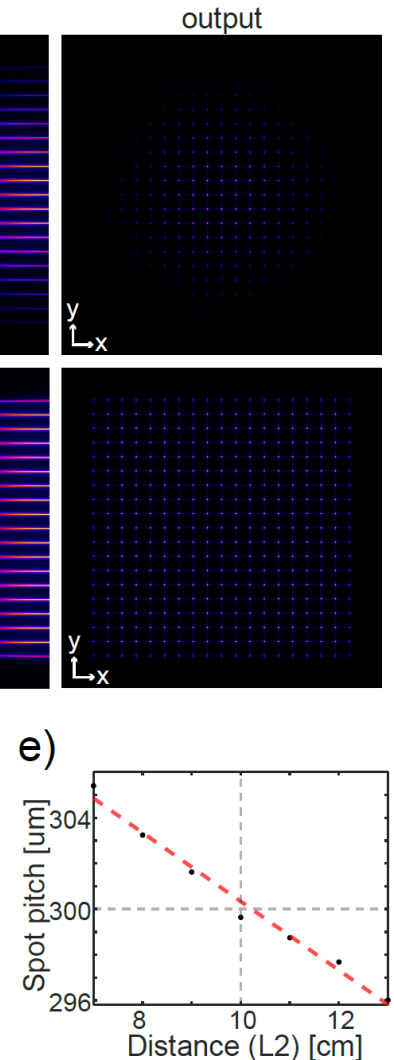

h)

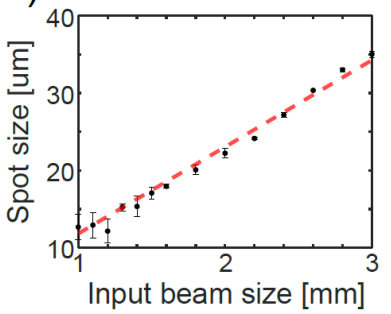

i) Collimating lens Flat-fielding MLAs

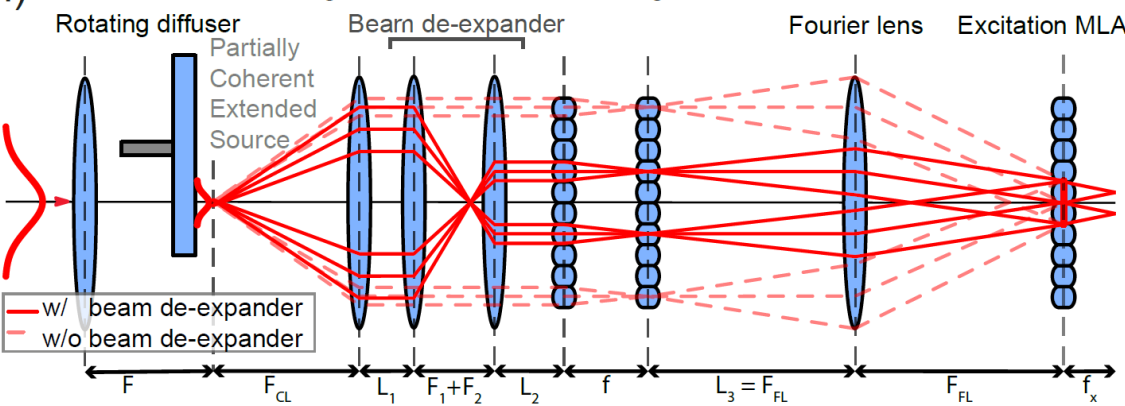

Figure 1 - Design and features of the mfFIFI module. a,b) Wave optics simulation with (a) a Gaussian beam or (b) a flat-top beam used as input onto the excitation MLA, showing their propagation and their effect on the output intensity of the generated excitation spots. c,d) Experimental positions of the excitation spots (blue dots) overlaid on a conjugate pinhole array (black circles) and the displacement between the two (yellow arrows) for (c) 
non-telecentric and (d) telecentric illumination of the excitation MLA reveals a change in the pitch of excitation spots caused by non-telecentric illumination. Data are from separately acquired images of pinholes and excitation spots. e) Plot showing the dependence of the pitch of the excitation spots on the offset of the Fourier lens back focal plane to the second flat-fielding MLA. Dashed line represents zero offset on the x-axis and the ground truth set by the pitch of the MLA on the y-axis. f) Image of excitation spots generated with a Gaussian beam as input into the excitation MLA. Directly implementing the Koehler integrator as the input results in large excitation spots. g) Limiting the number of flat-fielding MLA channels used for beam homogenization partially recovers the size of the excitation spots as achieved using Gaussian excitation (a), while maintaining uniform intensity of the Koehler integrator (f). h) The spot size increases linearly with the diameter of the beam incident on the flat-fielding MLAs over the given range. i) Complete design and implementation of the extended Koehler integrator for multi-focal flat illumination for field independent imaging (mfFIFI).

Previously, multifocal excitation for the iSIM was generated by illuminating a microlens array (MLA) with a collimated Gaussian beam ${ }^{6,9}$. As expected, the nonhomogeneous beam profile results in excitation points whose intensity depends on their location, a result which is recapitulated in a wave optics simulation (Figure 1a) (Supplemental information). Alternatively, illuminating the excitation MLA with a flat-top beam - such as that produced by a Koehler integrator - results in an array of multifocal excitation spots of homogeneous intensity (Figure 1b). This can be achieved by placing the excitation MLA at the front focal plane of the Fourier lens of the Koehler integrator, where the flat-top is focused (Supplemental Figure 1a). However, if the incident wavefront is not flat, the non-telecentric illumination of the excitation MLA produced by this configuration alters the pitch of the excitation spots in the focal plane of the excitation MLA (Figure 1c). This periodicity - usually determined by the pitch of the excitation MLA - must match the periodicity of additional components that are typically placed in conjugate image planes of confocal microscopes, such as a pinhole array. In the case of a mismatch of array elements, the light will be gradually occluded and decrease in intensity away from the center of the optical path. To solve this problem and achieve telecentric illumination of the excitation MLA, we place the Fourier lens of the Koehler integrator one focal length away 
135 from the second flat-fielding $\mathrm{MLA}^{27}$, resulting in a flat wavefront and thereby ensuring that

136 the pitch of the excitation is conserved (Figure 1d, Supplemental Figure 1b). Additionally, 137 adjustment of the distance between the second flat-fielding MLA and the Fourier lens can 138 be used to finely adjust the resulting periodicity of the excitation spots to match that of the 139 other optical elements (Figure 1e, Supplemental Figure 2a-d). This feature, not shared by 140 refractive beam-shapers, allows us to optimize transmission efficiency.

Finally, in confocal microscopy and its variants such as iSIM, maximizing the 142 achievable resolution requires focusing the excitation light to a diffraction-limited spot on the 143 sample. This imposes a limitation on the spot size that should be generated in the excitation 144 path. However, implementing a traditional Koehler integrator in the excitation path would 145 produce large excitation spots due to the nature of the partially coherent extended source created by the rotating diffuser (Figure 1e-f, Supplemental Figure 1). In that case, the accessible improvement in resolution would not be based on diffraction-limited performance. A possible solution would be to introduce a pinhole array to mask the 149 excitation spots that are focused onto the sample, but at a significant cost to the 150 transmission efficiency.

In an alternative design, we find that - contrary to the typical Koehler integrator where illuminating a maximal number of flat-fielding microlenses is preferred ${ }^{24-26}$ 153 illuminating fewer microlenses offers a solution to control the excitation spot size and 154 ensure diffraction-limited excitation at the sample (Figure 19,h). Incorporating a beam 155 expander to contract the light incident on the flat-fielding MLAs (referred to as a beam 156 contractor henceforth, Figure 1i, Supplemental Figure 1c) allows us to tune the apparent 157 size and angular distribution of the extended source. This shrinks the size of the excitation 158 spots, while maintaining efficient light transmission and homogeneity across the excitation 159 illumination. This configuration, which we call multifocal flat illumination for field160 independent imaging (mfFIFI), meets all the above requirements for homogeneous multifocal excitation and is shown in Figure 1i. 
These requirements can be described by geometrical optics and are a direct

163 outcome of ray transfer matrix calculations (Supplemental information, Supplemental

164 Figure 2), but the large parameter space makes the design and optimization of the mfFIFI 165 module non-trivial. For example, beam contraction presents a trade-off between 166 homogeneity of the multifocal excitation and the ability to achieve diffraction-limited 167 excitation and requires careful optimization (Supplemental information, Supplemental 168 Figure $3 \mathrm{~h}$ ). To facilitate the optimization and choice of components for efficient mfFIFI, we 169 provide the main design equations and an extended version of our existing wave optics 170 simulation platform ${ }^{10}$ (Supplemental information, Supplemental Figure 3, Supplemental 171 Table 1).

We tested the performance of the mfFIFI module when integrated into the excitation path of an $\operatorname{iSIM}^{14,31}$ (Supplemental information, Supplemental Figure 4). To visualize the excitation illumination of the iSIM, we used a concentrated dye solution ${ }^{28}$, and imaged the emitted light onto the camera without scanning (Supplemental information, 177 Supplemental Figure 4a). Using an approximately Gaussian beam $\left(\mathrm{M}^{2}<1.1\right.$, FWHM 178 diameter $\sim 12 \mathrm{~mm}$ ) to create the excitation pattern, as used in the initial iSIM design ${ }^{9,29}$, 179 results in excitation points of spatially varying intensity closely following a Gaussian 180 distribution (Figure 2a), as expected from the simulation (Figure 1a). Scanning the 181 Gaussian excitation points partially homogenizes the excitation along the scan direction; 182 however, the resulting illumination features a bright central region and strong roll-off away 183 from the central optical axis (Supplemental information, Supplemental Figure 5a,b). In 184 comparison, generating the excitation using the mfFIFI module results in a more uniform 185 multifocal excitation covering an area of approximately $100 \times 100 \mu \mathrm{m}^{2}$ (Figure $\mathbf{2 b}$ ), and a 186 more homogeneous excitation during scanning (Supplemental information, 187 Supplemental Figure 5c-e). 

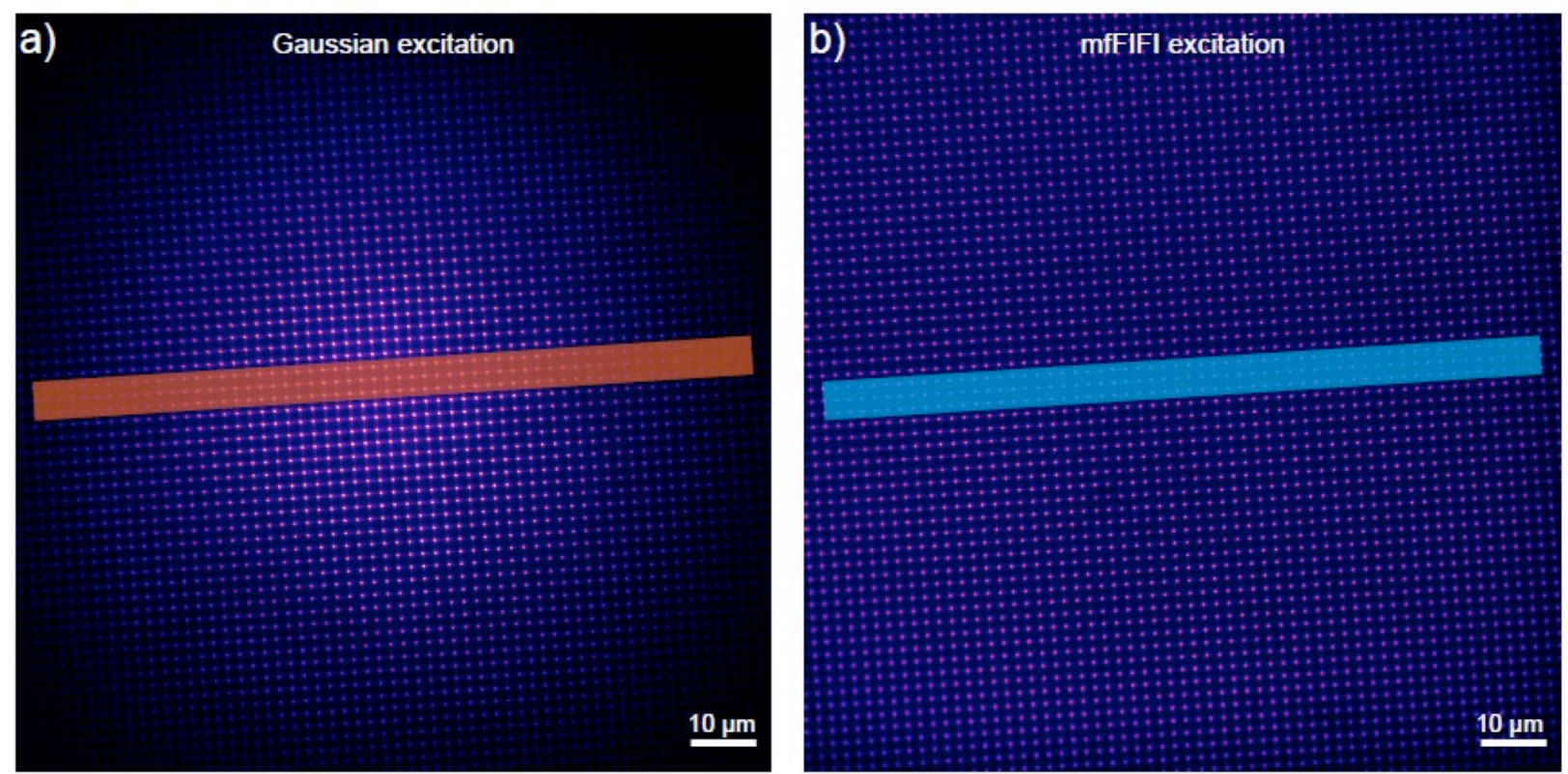

c)

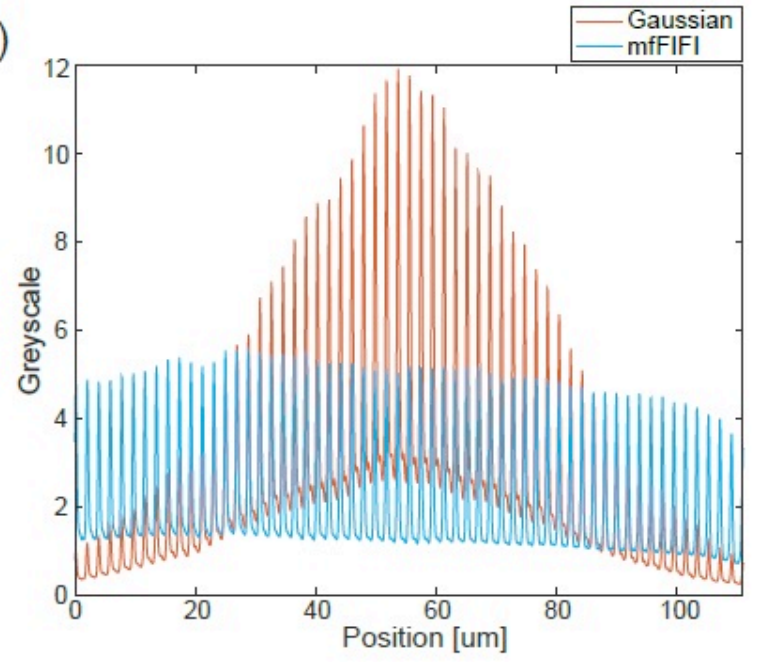

d)
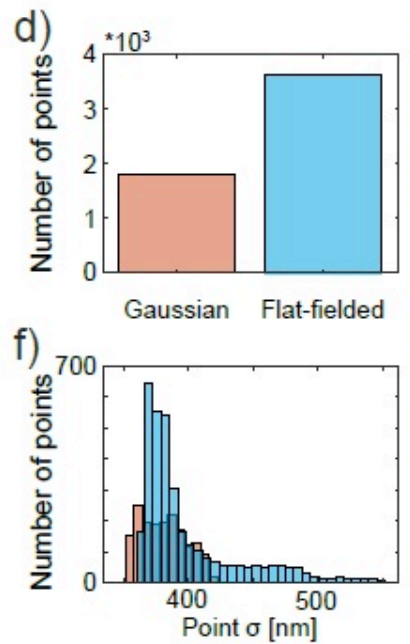
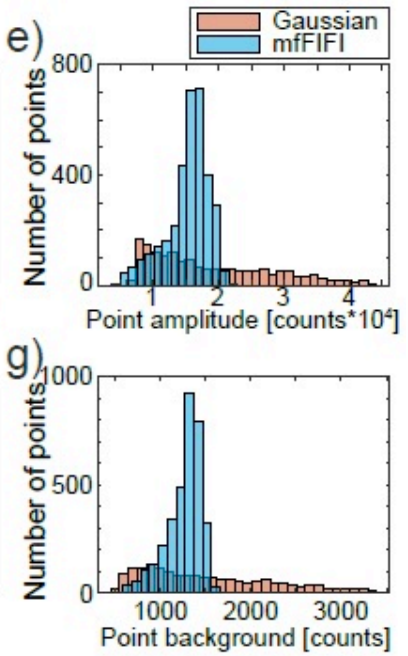

Figure 2 - Performance comparison between Gaussian and mfFIFI excitation. a,b)

190 Excitation points imaged onto a NaFITC fluorescent dye sample with (a) Gaussian and (b) mfFIFI excitation. c) Intensity profiles measured along lines in (a, b). d) Number of excitation points with a signal-to-noise ratio (SNR) above 3 in Gaussian and mfFIFI excitation. mfFIFI increases the number of excitation points by a factor of two. e-g) Histograms of excitation point (e) amplitudes, (f) variance and (g) background levels for Gaussian and mfFIFI excitation.

Comparing intensity profiles along the rows of excitation spots shows that mfFIFI 197 efficiently redistributes the spatially varying input Gaussian excitation over a larger area 198 (Figure 2c). Quantifying these differences, we find that mfFIFI doubles the number of excitation spots above background levels compared to the Gaussian excitation, while resulting in a much narrower distribution of spot amplitudes and background (Figure 2d-e, 
201 g). Furthermore, this improvement comes at no measurable cost to the quality of the

202 structured illumination. We find that both the size of the excitation spots required for

203 resolution improvement in confocal microscopy (Figure 2f) and their periodicity $(222.3 \pm 0.3$

$204 \mu \mathrm{m}$ (Gaussian) and 222.2 $\pm 0.3 \mu \mathrm{m}$ (mfFIFI) (mean \pm S.D.)) are maintained, and compare

205 well with the ground truth (222 $\mu$ m excitation MLA pitch) (Supplemental information). This

206 was further verified by imaging $100 \mathrm{~nm}$ fluorescent beads, showing comparable lateral 207 resolution between mfFIFI (214 $\pm 5 \mathrm{~nm}$, mean \pm S.D.) and Gaussian illumination (219 \pm 12

$208 \mathrm{~nm}$ ), based on FWHM measured on raw iSIM data before deconvolution, or $\sim 140 \mathrm{~nm}$ lateral 209 and $\sim 350 \mathrm{~nm}$ axial resolution after deconvolution ${ }^{9}$ (Supplemental information).

\section{Large FOV iSIM imaging and FOV stitching}

We used our custom-built iSIM setup as a platform to combine the capability of

212 mfFIFI excitation with a scientific complementary metal-oxide semiconductor (sCMOS)

213 camera for detection to perform large FOV volumetric imaging at doubled resolution. While

214 existing commercial SIM setups are mostly limited to FOVs with a linear dimension of 30-60

$215 \mu \mathrm{m}$, our setup reaches $>100 \times 100 \mu \mathrm{m}^{2}$, thus providing a $\sim 4$ - to 10 -fold increase in FOV area.

216 We used mfFIFI iSIM to simultaneously image multiple mammalian cells within a single

217 FOV at doubled resolution (Figure 3a, Supplemental Movie 1). Accessible imaging 218 speeds are limited merely by the frame rate of the detector; thus, frame rates of up to 100 $219 \mathrm{~Hz}$ as reported for $\operatorname{iSIM}^{9,29}$ are preserved, since the increase in FOV is achieved by 220 improved parallelization from the increased number of illumination spots. Thus, the increase 221 in FOV translates directly into an increase in throughput. 

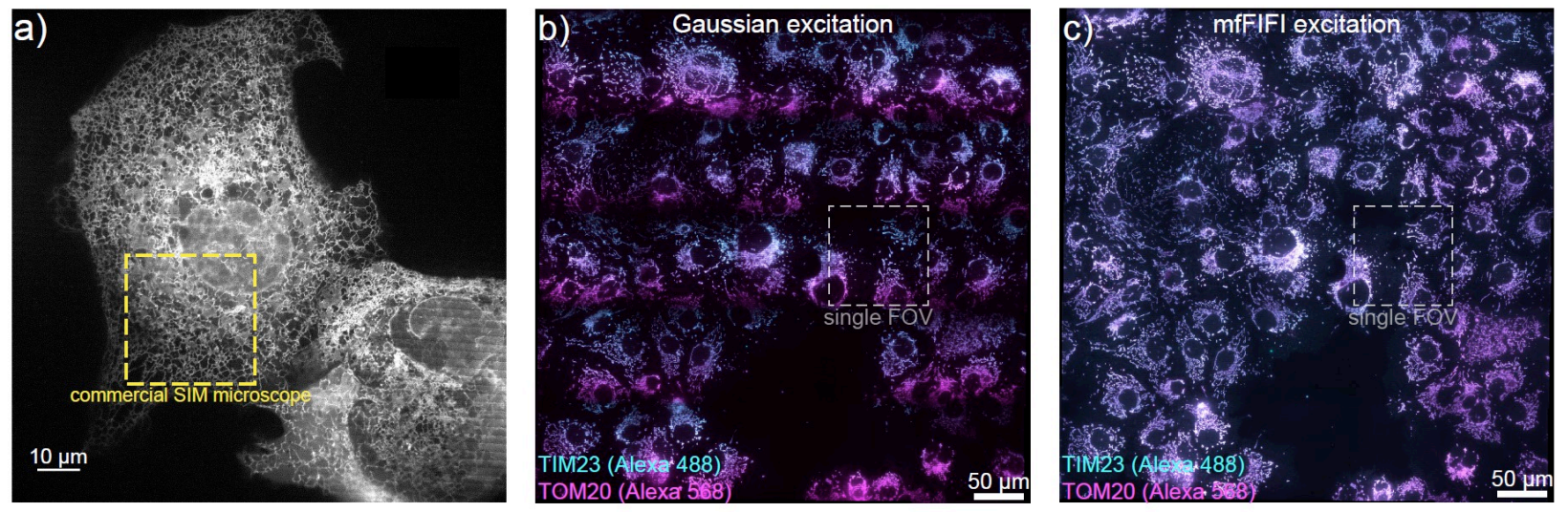

d)

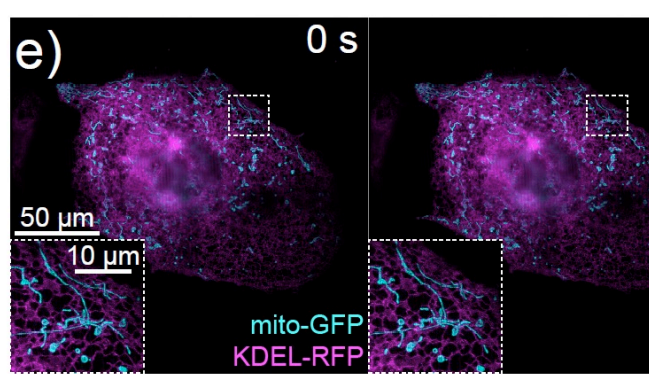

\section{$4 \mathrm{~s}$}

single FOV

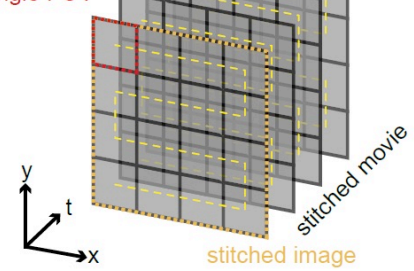

Figure 3 - Large FOV imaging and multi-FOV stitching. a) Cos7 cell expressing KDELRFP imaged with an iSIM using mfFIFI excitation and an sCMOS camera enabling imaging of FOVs up to $\sim 115 \times 115 \mu \mathrm{m}^{2}$ at doubled resolution compared to diffraction limited performance. Yellow square shows the size of a $30 \times 30 \mu \mathrm{m}^{2} \mathrm{FOV}$ available on a standard commercial SIM setup. Full movie shown in Supplemental Movie 1. b,c) Raw iSIM images of Cos7 cells immunolabelled for TIM23 and TOM20, stitched from a 5x5 grid of FOVs using

(b) Gaussian and

(c) mfFIFI excitation.

d) Introducing an additional layer of

231 parallelization into iSIM imaging by iterating over multiple FOVs allows trading off the speed

232 of iSIM imaging to construct larger FOVs at high speed and doubled resolution compared to

233 diffraction limited performance. e) Montage showing frames from a high-speed movie built 234 up over a $2 \times 2$ grid of FOVs (Supplemental information, original movie $2 \mathrm{~s}$ temporal resolution) of cells expressing mito-GFP and KDEL-RFP. Full movie shown in Supplemental Movie 2.

The effective image size can be further extended by stitching together adjacent FOVs. Such an approach requires homogeneous illumination to allow seamless stitching (Figure 3b). The speed of iSIM imaging combined with the large FOV enabled by mfFIFI

240 allowed us to stitch together dual-color 3D stacks covering a $500 \times 500 \times 5 \mu \mathrm{m}^{3}$ volume with a $2415 \times 5$ grid of FOVs, each acquired within 5 seconds. With this approach, within 2 minutes, we 242 could capture 3D images of more than 80 cells stained for the mitochondrial inner (TIM23) 
243 and outer (TOM20) membrane translocases, revealing coupled geometries of the two

244 membranes (Figure 3b-c). The current limiting factor for multi-FOV imaging is the speed of

245 stage translation and synchronization between stage translation and the rest of the iSIM

246 acquisition control (Supplemental information). In contrast, the same acquisition without

247 mfFIFI resulted in varying intensity across each FOV, with pronounced dark boundaries at

248 the borders of individual FOVs (Figure 3b).

249 For time-lapse imaging, we introduced an additional layer of parallelization by

250 iterating the acquisition procedure over a grid of FOVs (Figure 3d). By trading temporal

251 resolution for a gain in imaging area, we can use the speed of iSIM imaging to cover

252 multiple FOVs. For example, by iterating over a 2x2 grid of FOVs we could capture four

253 time more regions of close contact between mitochondria and ER, known to serve as

254 hotspots for mitochondrial division ${ }^{30}$ at a temporal resolution of 2 seconds (Figure $3 \mathbf{e}$,

255 Supplemental Movie 2) (Supplemental information). This illustrates how processes with

256 less demanding temporal dynamics can benefit from mfFIFI to extend the throughput of

257 iSIM without compromising spatial resolution.

258 High-throughput super-resolution imaging of expanded centrioles

A recently developed variant of ExM termed ultrastructure expansion microscopy

$260(\mathrm{U}-\mathrm{ExM})^{31}$ enables preservation of the structure and molecular identity of multi-protein 261 assemblies (particles). When combined with iSIM imaging, an expansion factor of $\sim 4$ 262 results in an effective resolution of $\sim 140 / 4=35 \mathrm{~nm}$ laterally and $\sim 350 / 4 \approx 90 \mathrm{~nm}$ axially 263 after deconvolution. Compared with current state-of-the-art super-resolution microscopes 264 with similar resolution performance, such as the HT-STORM ${ }^{10}$ capable of imaging a $265100 \times 100 \mu \mathrm{m}^{2}$ FOV in $\sim 5-10$ minutes or a similar FOV with easySLM-STED ${ }^{32}$ in $60-80$ 266 minutes, the iSIM is capable of stitching together images of expanded samples to form an 267 equivalent FOV within 2-5 seconds. This represents a 100-1000-fold improvement in imaging throughput. Therefore, acquiring datasets of thousands of particles that would take 
weeks or months to acquire on a conventional STORM or STED microscope require only 1-

2702 hours on the high-throughput iSIM.

As a proof-of-concept for iSIM/U-ExM, we set out to analyze centrioles, organelles

272 found in most eukaryotic cells that seed the formation of the axoneme in cilia and the

273 centrosome in animal cells. Centrioles have a characteristic nine-fold radial symmetric

274 arrangement of microtubule triplets towards the proximal end of the organelle, composed of

275 complete A-microtubules and incomplete B- and C-tubules ${ }^{33}$, with a transition to A- and B-

276 microtubule doublets towards the centriole distal end ${ }^{34}$ (Figure 4a). It is known that

277 centriolar tubulin is enriched in post-translational modifications (PTMs), including

278 acetylation and polyglutamylation ${ }^{35,36}$. Acetylation is known to stabilize microtubules,

279 increase their flexibility and protect them against mechanical aging ${ }^{37-39}$. Glutamylation has

280 been postulated to likewise stabilize microtubules in the centriole and protect the organelle

281 from pulling and pushing forces acting during mitosis ${ }^{36,40,41}$. Although these observations

282 indicate that centrioles might be stabilized via tubulin acetylation and polyglutamylation, the

283 spatial organization of these PTMs on the organelle remains largely unexplored.

To analyze human centrioles in a similar stage of the cell cycle and of the centriole duplication cycle, we synchronized RPE-1 cells with thymidine to arrest them at the G1/S transition and with the Plk4 inhibitor Centrinone to prevent the formation of new centrioles ${ }^{52}$.

287 After expansion and staining with antibodies against acetylated and polyglutamylated 288 tubulin, we imaged $>100$ centrioles in 3D in their cellular context per hour, revealing the 289 distribution of these PTMs along the organelle. Analyzing over 400 individual particles 290 extracted from these images (Supplemental Figure 6) revealed that centriolar tubulin 291 appears uniformly acetylated, while polyglutamylation terminates $\sim 30 \mathrm{~nm}$ before the distal 292 end (Figure 4b, Supplemental Figure 7a-c). Moreover, the polyglutamylation signal 293 exhibits a larger diameter (Figure 4b, Supplemental Figure 7a-c), in line with the fact that 294 acetylation occurs witin the microtubule wall and polyglutamylation on the outer surface of 295 the polymer ${ }^{31}$. 


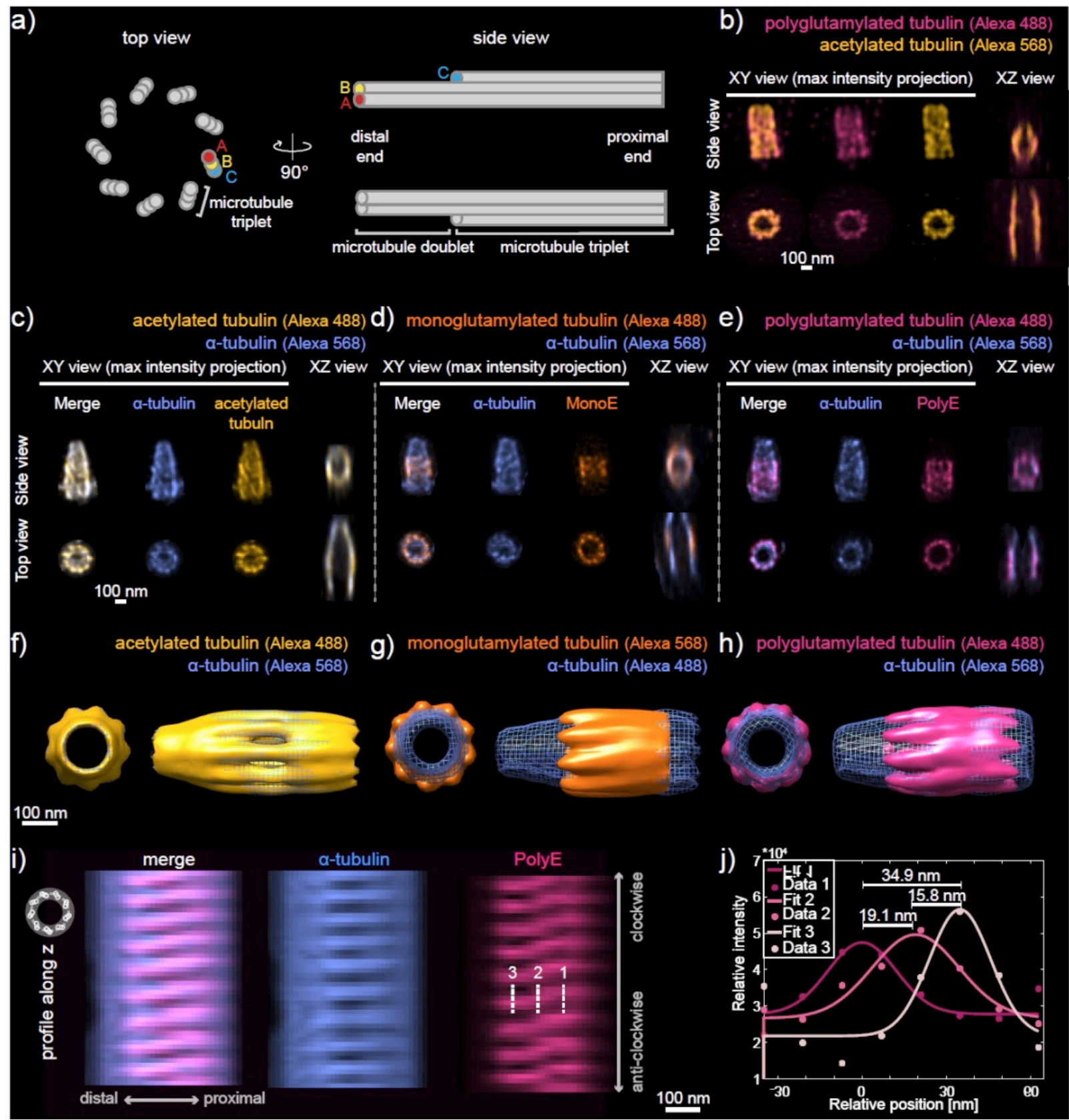

Figure 4 - High-throughput super-resolution imaging of expanded centrioles for mapping post-translational modifications of centriolar tubulin. a) Schematic representation of the centriolar microtubule wall viewed from the side (left) and top from the distal end. The A-, B- and C-microtubules are indicated. b) Examples of individual mature human centriole particles orthogonal (side view) or parallel (top view) to the optical axis collected in expanded RPE-1 cells labelled for acetylated tubulin and polyglutamylation (PolyE). The lateral $(X Y)$ maximum intensity projection and axial $(X Z)$ cross sections are shown. c-e) Examples of individual purified Chlamydomonas reinhardtii centriole particles viewed orthogonal (side view) or parallel (top view) to the optical axis labelled for $\alpha$-tubulin as reference and (c) acetylated tubulin, (d) monoglutamylated tubulin (GT335, MonoE) and (e) polyglutamylated tubulin (PolyE). f-h) High resolution particle averaging reconstructions 

of (f) acetylated tubulin, (g) MonoE and (h) PolyE with $\alpha$-tubulin as reference. Scale bars: $100 \mathrm{~nm}$ (pre-expansion). Top views shown from the distal end. i) Circular axial projection (XZ profile) of the centriole reconstruction from (h) showing a-tubulin and PolyE signal around the centriole (viewed from the outside). j) Intensity profiles measured along the dashed lines from (i) showing the radial displacement of the PolyE signal.

To further increase throughput, we sought to expand purified centrioles to capture dozens of pairs within a single FOV rather than just one per cell. We used centrioles purified from Chlamydomonas reinhardtii to this end since the U-ExM protocol had been optimized for this species ${ }^{31}$. Similarly to what we observed with human centrioles in the cellular context, we observe that in centrioles purified from Chlamydomonas reinhardtii, acetylated tubulin colocalizes with the microtubule wall along the entire length of the organelle (Figure 4c), in agreement with previous reports ${ }^{42}$. In contrast, we found that tubulin monoglutamylation (MonoE) is concentrated in the central core region of Chlamydomonas reinhardtii centrioles (Figure $4 \mathbf{d}$ ), as previously reported ${ }^{42}$. Since MonoE recognizes the first glutamylation branching required for polyglutamylation (PolyE), we would expect the distribution of PolyE to partially or completely overlap with that of MonoE. Surprisingly, we found that although PolyE also localizes on the outer surface of the microtubule triplets where it has been suggested to localize to the C-microtubule ${ }^{31}$, it covers a wider band along the length axis than MonoE (Figure 4e, Supplemental Figure 7, 12). This difference could reflect lower MonoE antibody labelling efficiency, since saturating the signal shows similar MonoE/PolyE localization.

The high throughput offered by mfFIFI-iSIM allowed us to collect 3D images of thousands of purified expanded Chlamydomonas reinhardtii centrioles per hour (Supplemental Figure 8, Supplemental Table 2). Having obtained 3D rather than 2D particles allowed us to use fewer particles to perform averaging and high-resolution multicolor reconstruction ${ }^{44}$ compared to previous approaches using STORM $^{45}$ or electron microscopy ${ }^{46,47}$. Thus, we classified particles before reconstruction to identify and average only those particles sharing a high degree of similarity (ranging from $10-50 \%$ of the whole dataset), and thereby achieve even higher resolution reconstructions (Figure 4f-h, 
337 Supplemental information, Supplemental Figure 9). Here, we have focused on the most

338 frequently appearing class, but other classes could also be independently reconstructed.

339 For mapping multiple PTMs within the centriole, we employ dual-color labeling, using $\alpha$ -

340 tubulin staining of the microtubule triplet wall as a reference to subsequently align all PTMs

341 (Supplemental Figure 10).

The resulting reconstructions revealed an unexpected shift in the tangential position

343 of the PolyE signal from the proximal to the distal end (Figure 4h), which is also sometimes

344 visible in individual raw particles (Supplemental Figure 8b inset, Figure 4e). To account

345 for this shift, we considered two possibilities: 1) the whole microtubule triplet twists around

346 its axis while the PolyE signal remains localized to the C-microtubule ${ }^{48}$ giving it an apparent

347 twist, and 2) the PolyE signal changes microtubule localization within the microtubule triplet.

348 Arguing against the first possibility, the microtubule triplet twists only within $\sim 100 \mathrm{~nm}$ of the

349 proximal end (Le Guennec et al., in press), while in our reconstruction the PTM twist occurs

350 within a wider region covering $\sim 250 \mathrm{~nm}$ from the proximal end,. Moreover, we determined

351 that the first possibility would be expected to cause a moderate $\sim 6 \mathrm{~nm}$ shift of the PolyE

352 signal, and the second a more pronounced $\sim 35-40 \mathrm{~nm}$ shift (Supplemental information,

353 Supplemental Figure 11). From our reconstruction, we measure a twist of $\sim 34.9 \mathrm{~nm}$,

354 which cannot be explained purely by twisting of the microtubule triplet (Figure $4 \mathbf{i}, \mathbf{j}$,

355 Supplemental information, Supplemental Figure 11). Therefore, we propose that 356 polyglutamylation changes localization within the microtubule triplet along the centriole,

357 moving from A- on the proximal end to the C-microtubule more distally.

\section{Conclusion}

To summarize, we designed a flat-fielding method for efficient multifocal excitation, accompanied by a wave optics simulator which ensures optimal resolution performance. While the Koehler integrator has spot generating properties when used with coherent illumination, it introduces a strong wavelength dependence of the periodicity, making it 363 unsuitable as such for multi-color applications ${ }^{26}$. mfFIFI provides a largely wavelength- 
364 independent solution for generating homogeneous multi-focal excitation, while benefiting

365 from the homogenizing properties of the Koehler integrator. We implemented the mfFIFI

366 module into an iSIM microscope, since its fast acquisition speeds and resolution doubling

367 give it good potential for high-throughput super-resolution imaging. However, the mfFIFI

368 module could be extended to any multi-focal excitation microscope such as a spinning disk

369 confocal microscope ${ }^{6}$ using a Nipkow disk configuration, but not with other flat-fielding

370 solutions using diffractive optical elements ${ }^{20}$, spatial light modulators (SLM) ${ }^{21}$ or beam

371 splitters ${ }^{23}$. Furthermore, combining mfFIFI with phase masks required for donut beam

372 shaping could extend its application to parallelized STED and RESOLFT microscopes.

373 Flat-fielding enables multi-field of view imaging, which is a powerful way to maintain

374 resolution while increasing the effective image size to span more length scales. This is

375 important for sample spanning multiple fields of view, as in the case of tissues, or expanded

376 samples. ExM sacrifices the effective FOV to increase resolution, and stitching provides a

377 way to maintain throughput, allowing cellular features or structures to be imaged in situ, as

378 we demonstrate by imaging hundreds of human centrioles in cells.

Particle averaging and reconstruction is traditionally used in ultrastructural biology,

and leverages large datasets to capture the intrinsic variability within particles through classification before reconstruction, while averaging out the noise. In the case of ExM, different gels can have slightly different expansion factors, but this issue is circumvented by our iSIM setup since thousands of particles can be acquired from a single gel section ( $5 \times 5$ $384 \mathrm{~mm}^{2}$ ). In our case, the collected datasets were sufficiently large to allow particle 385 classification prior to averaging, an approach which improves the resolution of the 386 reconstruction and which, to our knowledge, has not been previously used for fluorescence 387 super-resolution. In addition to a 100-1000-fold improvement in throughput of iSIM/U-ExM 388 over HT-STORM ${ }^{10,45}$ or easySLM-STED ${ }^{32}$, expanded samples offer enhanced accessibility 389 of antibodies to bind proteins in crowded assemblies. Beyond particle averaging, high390 content or machine learning approaches rely on large datasets for training or screening, 391 and can benefit from the combination of throughput and resolution described here. 
Our previous attempts to image centriolar microtubules with STORM proved largely

393 unsuccessful, restricting previous studies to the more accessible epitopes ${ }^{45}$. Here, we

394 reveal previously unobserved molecular organization, showing high coverage of

395 polyglutamylation in human centrioles, as well as precise localization to microtubule triplets

396 along the Chlamydomonas reinhardtii centriole. We find that polyglutamylation appears to

397 shift from the A-microtubule proximally to the C-microtubule more distally. It is possible that

398 such a glutamylation shift reflects a centriolar tubule code that defines the spatial

399 boundaries of the various elements that compose it, which could determine the positioning

400 of different structural elements along the length of the organelle. 
Methods

Sample preparation

Cos-7 and RPE-1 cells were grown in Dulbecco's modified Eagle medium (DMEM) supplemented with $10 \%$ fetal bovine serum (FBS). Cells were plated on $25 \mathrm{~mm}$, \#1.5 glass coverslips (Menzel) 16-24 h prior to transfection or fixation at a confluency of $\sim 10^{5}$ cells per

407 well.

Transfections using [insert], were performed with Lipofectamine 2000 (Life

409 Technologies) using $150 \mathrm{ng}$ of [insert] and $1.5 \mu \mathrm{L}$ of the Lipofectamine 2000 reagent in 100 $410 \mu \mathrm{L}$ Opti-MEM medium per 6 well.

For synchronization of RPE-1 cells at the G1/S transition and the onset of centriole assembly, $\sim 125^{\prime} 000$ cells were seeded for $24 \mathrm{~h}$ in six well-plates (Merck, TPP, 92006) on

$41312 \mathrm{~mm}$ coverslips in DMEM [insert]. Thymidine (Merck, T1895, $1 \mathrm{mM}$ ) and Centrinone 414 (Lucerna-Chem, MCE-HY-18682, $300 \mathrm{nM}$ ) were added for $18 \mathrm{~h}$, before fixation of cells with $415-20^{\circ} \mathrm{C}$ methanol for $5^{\prime}$.

For immunofluorescence experiments with COS-7 cells, cells were washed in pre-

417 warmed PBS before being fixed in pre-warmed fixation buffer (4\% paraformaldehyde in 418 PBS). Cells were then permeabilized in $0.25 \%$ Triton-X in PBS for 10 min. After washing in 419 PBS for 5 min, cells were incubated in blocking buffer (1\% BSA in PBS) for 60 min. The 420 primary antibodies (Tom20-rabbit (1:50, FL-145 sc-11415 Santa Cruz Biotechnologies), 421 Tim23-mouse (1:100, 611222 BD Biosciences)) diluted in blocking buffer were incubated 422 for 60 min before washing 3 times in $0.2 \%$ BSA with $0.25 \%$ Triton-X in PBS for 10 min. 423 Secondary antibodies (AlexaFluor 488 goat anti-mouse IgG $(H+L)(1: 150, \quad A 28175$

424 ThermoFisher), AlexaFluor 568 goat anti-rabbit $\lg G(H+L)(1: 150$, A11011 ThermoFisher $))$ 425 were diluted in blocking buffer before incubation for another $30 \mathrm{~min}$. The sample was 426 incubated in the dark, then washed 3 times with PBS before imaging. 
The iSIM setup was partly based on previously described implementations ${ }^{9,29}$. Two lasers with wavelengths of $488 \mathrm{~nm}$ (Sapphire 488-300 CW CDRH, Coherent) and $561 \mathrm{~nm}$ (gem 561, Laser Quantum $\mathrm{GmbH}$ ) were combined using a dichroic mirror (F48-486, Analysentechnik) and controlled through an acousto-optic tunable filter (AOTFnC-400.650TN, AA Optoelectronic). In the case of Gaussian excitation, the beam was expanded with a 10x beam expander ( $f_{1 a}=40 \mathrm{~mm}, f_{2 a}=400 \mathrm{~mm}$, Thorlabs). In the mfFIFI path, a focusing 434 lens $\left(\mathrm{f}_{\mathrm{FC}}=50 \mathrm{~mm}\right)$ was used to focus the light near a rotating diffuser $\left(2.5^{\circ} \pm 0.25^{\circ} \mathrm{FWHM}\right.$ at $650 \mathrm{~nm}, 24-00066$, Süss MicroOptics SA) before collimation by a collimating lens $\left(\mathrm{f}_{\mathrm{CL}}=\right.$ $60 \mathrm{~mm}$, Thorlabs). The collimated beam was contracted by a factor of 4 by two lenses $\left(\mathrm{f}_{1 \mathrm{~b}}=\right.$ $437120 \mathrm{~mm}, \mathrm{f}_{2 b}=30 \mathrm{~mm}$, Thorlabs) before illuminating two flat-fielding MLAs (300 $\mu \mathrm{m}$ pitch, $43810 \mathrm{~mm} \times 10 \mathrm{~mm}, \mathrm{f}=4.78 \mathrm{~mm}$, square lenses, 18-00157, Süss MicroOptics SA). The flat 439 field was then focused by a Fourier lens $\left(f_{F L}=300 \mathrm{~mm}\right.$, Thorlabs). Both paths then 440 illuminated the excitation MLA (222 $\mu \mathrm{m}$ pitch, 1" diameter, $f=6 \mathrm{~mm}$, square lenses APO-Q441 P222-R2.74, Advanced Microoptic Systems $\mathrm{GmbH}$ ). The excitation was relayed by scan 442 lenses ( $f_{S L}=190 \mathrm{~mm}, 55-S 190-60-V I S$, Special Optics) to and from the scanning mirror 443 (SPO9086 Rev B Coated X Mirror, Sierra Precision Optics) mounted on a galvanometer 444 scanner (QS-12, N-2071, Nutfield Technology). The excitation was then imaged onto the 445 sample using a tube lens ( $f_{\mathrm{TL}}=350 \mathrm{~mm}, 49-289-\mathrm{INK}$, Edmund Optics) and an objective lens 446 (APON60XOTIRF, Olympus), resulting in a final magnification of $\sim 116 x$. The sample was 447 placed on a precision aligned microscopy platform including a micropositioning control 448 (RM21-AZ-AXY-RMS-M, Mad City Labs) and a Z piezo stage (Nano-Z200, Mad City Labs). 449 The emission was relayed back through the excitation side of the scanning mirror and split 450 using a dichroic mirror (F58-488S, Semrock). The emission was then pinholed with a 451 pinhole array (chrome on 0.090-inch-thick quartz, $222 \mu \mathrm{m}$ pitch, $40 \mu \mathrm{m}$ diameter, 452 Photosciences) and relayed by two relay lenses ( $f_{R}=300 \mathrm{~mm}$, Thorlabs) before contracting 453 each emission spot by a factor of 2 using another MLA (222 $\mu \mathrm{m}$ pitch, 1 " diameter, $f=0.93$ 
$454 \mathrm{~mm}$, square lenses APO-Q-P222-R0.425, Advanced Microoptic Systems GmbH). The

455 emission was then relayed using scan lenses on the emission side of the scan mirror,

456 towards a filter wheel (Lambda 10-B, Sutter Instruments, Science Products) with 2 notch

457 filters (NF-03-488E-25 and NF-03-561E-25, Semrock) mounted to block the excitation

458 wavelengths. The transmitted emission was then collected by a sCMOS camera (PrimeBSI,

459 01-PRIME-BSI-R-M-16-C, Photometrics). The size of a square camera pixel corresponds to

$46056 \mathrm{~nm}$ on the sample.

461 The microscope was controlled using a custom written MATLAB script which

462 controlled an analog output card (PCI-6733, National Instruments) and breakout box (BNC-

4632110 , National Instruments) for precise control and synchronization of the scan mirror, 464 AOTF, camera and Z-stage. The XY stage and camera were controlled through 465 MicroManager ${ }^{49}$.

For live cell imaging, imaging was performed at $37^{\circ} \mathrm{C}$ in pre-warmed Leibovitz 467 medium in a top stage incubator (H301-PRIOR-NZ100-H117, Okolab). Imaging of 468 immunostained samples was performed in PBS. Expanded samples were mounted as 469 previously described ${ }^{31}$. Briefly, the expansion factor was determined before imaging by 470 measuring the gel size with a caliper (precision $\pm 0.01 \mathrm{~mm}$ ). The gel was then cut using a 471 razor, before removing excess water and placing the gel on a poly-D-lysine treated 472 coverslip (25 mm round coverslips, Mendel \#1.5) already placed inside the imaging 473 chamber. After gently pressing on the gel to ensure attachment to the coverslip, a few

474 drops of $\mathrm{ddH}_{2} \mathrm{O}$ were added on top of the sample to avoid shrinkage during imaging.

475 Image acquisition was performed using a custom written MATLAB script, in combination 476 with MicroManager ${ }^{49}$.

\section{7 iSIM deconvolution}

Raw iSIM images were deconvolved using the Lucy-Richardson deconvolution

479 algorithm implemented in MATLAB and provided by Dr. Hari Shroff ${ }^{9}$. Each raw z-stack was 480 deconvolved for 40 iterations. 


\section{mfFIFI characterization platform}

An optical characterization setup was built to measure the variation in pitch and spot size of the multifocal points. A laser beam with a wavelength of $640 \mathrm{~nm}$ (CUBE 640-100C, Coherent) was expanded with a $6 x$ beam expander ( $f=50 \mathrm{~mm}, f=300 \mathrm{~mm}$, Thorlabs) with a pinhole (P50D, Thorlabs) at the joint focal plane of the two lenses to spatially filter the beam. The beam is passed through a Köhler integrator, made of the following components:

487 focusing lens $\left(f=80 \mathrm{~mm}\right.$, Thorlabs), rotating diffuser $\left(2.5^{\circ} \pm 0.25^{\circ} \mathrm{FWHM}\right.$ at $650 \mathrm{~nm}, 24-$ 488 00066, Süss MicroOptics SA), collimating lens ( $f=40 \mathrm{~mm}$, Thorlabs), two flat-fielding MLAs 489 (300 $\mu \mathrm{m}$ pitch, $10 \mathrm{~mm} \times 10 \mathrm{~mm}, \mathrm{f}=4.78 \mathrm{~mm}$, square lenses, 18-00157, Süss MicroOptics SA), Fourier lens ( $f_{F L}=300 \mathrm{~mm}$, Thorlabs). An iris (ID25, Thorlabs) is used before the two MLAs to control the portion of the beam which is allowed to propagate forward to reach and illuminate MLAs, by adjusting the opening diameter of the iris. An excitation MLA (300 $\mu \mathrm{m}$ 493 pitch, $10 \mathrm{~mm} \times 10 \mathrm{~mm}, \mathrm{f}=8.72 \mathrm{~mm}$, square lenses, 18-00221, Süss MicroOptics SA) is 494 used to produce the multifocal points. The size of the multifocal illumination is reduced by a 495 factor of $0.6 x$ by a beam de-expander $(f=50 \mathrm{~mm}, f=30 \mathrm{~mm}$, Thorlabs) to be of appropriate size for the sensor of the camera (DCC1545M, Thorlabs).

To obtain the data shown in Figure $1 \mathrm{e}, \mathrm{h}$, we used the characterization platform to test the parameters affecting the spot size and pitch. A Vernier caliper was used to measure the iris opening diameter, which was varied methodically to control the size of the

500 beam which illuminates the MLAs. Images of the beam at different iris opening diameters 501 were analyzed using a MATLAB script, which allowed us to fit each excitation point to a 2D 502 Gaussian and measure the multifocal points' mean spot FWHM as function of iris diameter. 503 In order to study the variation in the pitch of the multifocal points, the distance between the 504 Fourier lens and the second flat-fielding MLA was varied by displacing the flat-fielding 505 MLAs. A MATLAB script is used to measure the mean pitch at each distance, so that the 506 relationship between this distance and the pitch can be determined. 


\section{Image analysis}

\section{Plateau uniformity quantification}

The homogeneity was quantified using the plateau uniformity definition based on the

510 FWHM of the histogram of the intensity values, according to ISO, ISO 13694:2018: Optics

511 and optical instruments - lasers and laser-related equipment - test methods for laser beam

512 power (energy) density distribution.

\section{Illumination profile measurement}

The illumination profiles used to optimize the pitch (telecentricity) and spot size of

515 the multifocal excitation were visualized using a small color CMOS camera (DCC1645C,

516 Thorlabs), placed in an intermediate image plane of the iSIM, between the second scan

517 lens and tube lens, to avoid introducing other aberrations along the whole optical path of

518 the iSIM.

The excitation profile at the sample was measured using a highly concentrated

520 fluorescent dye sample ${ }^{32}$, as previously described ${ }^{9}$. Briefly, sodium fluorescein (NaFITC)

521 powder was diluted in deionized water, followed by vortexing and sonication until the

522 powder was completely dissolved. A $10 \mu \mathrm{L}$ drop was placed on a $25 \mathrm{~mm}$ coverslip, before

523 covering with a $12 \mathrm{~mm}$ coverslip. The sample was then sealed using nail polish. The same

524 dye sample was used to measure both the $488 \mathrm{~nm}$ and $561 \mathrm{~nm}$ illumination profiles. The 525 illumination spots were characterized by fitting a 2D Gaussian to the intensity image. The 526 threshold was set so that no points in the background were detected. The scanning profiles 527 were measured with a 150 pixel thick line in Fiji50.

In the case of excitation spots visualized in Figure $1 \mathrm{f}, \mathrm{g}$ and the quantification in

529 Figure $1 \mathrm{e}, \mathrm{h}$, the illumination profile was imaged directly onto a camera placed in a 530 conjugate image plane. 
Bead FWHM analysis

To characterize the performance of the iSIM microscope, we used $100 \mathrm{~nm}$

534 Tetraspeck beads (ThermoFisher Scientific, T7279) deposited on poly-L-lysine treated 535 coverslips. Bead size was determined by fitting a Gaussian profile to the intensity profile of 536 the bead and extracting the FWHM.

\section{Centriole shape and coverage analysis}

To analyze the diameters of different PTM localizations within the centrioles, we measured the intensity profile through the centers of top view centrioles (central plane,

541 centriole barrel parallel to the imaging axis). The profile was measured using a 7 pixel thick 542 line in Fijij ${ }^{50}$. Each peak was fitted to a Gaussian profile to localize its center, before 543 calculating the diameter as the distance between the two peaks on opposing sides of the 544 centriole ring.

To analyze the PTM distribution along the length of the centriole and their coverage,

546 we measured the intensity profile along the length of the centriole using a 10 pixel thick line

547 and from summed intensity projections of side view centrioles (centriole barrel orthogonal to 548 the imaging axis). We then took the width of the profile at $1 / 4$ of the maximal signal as the 549 length, to be less susceptible to noise. The coverage was then calculated by dividing the 550 length of the PTM in question, by the length of the reference label (acetylated tubulin for 551 human centrioles and a-tubulin in the case of Chlamydomonas reinhardtii centrioles).

\section{$552 \quad$ Multiple field of view stitching}

For stitching of multiple images, raw images or z-stacks were acquired with a $10 \%$

554 overlap for both Gaussian and mfFIFI excitation. The acquired images were then combined using the Grid/Collection stitching tool in $\mathrm{Fiji}^{50}$ using the linear blending method with the 
556 default values of 0.30 for regression threshold, 2.50 for max/avg displacement threshold

557 and 3.50 for absolute displacement threshold.

\section{Wave optics simulations}

The mfFIFI simulation platform has been adapted based on previous work ${ }^{9}$. All simulations are based on standard numerical Fourier optics algorithms and rely on angular spectrum propagation methods to simulate the propagation of an optical wave. An initial

562 simulation showing the effect of illuminating the excitation MLA with Gaussian and flat-

563 fielded profiles was performed using phase masks to represent optical components.

564 The parameters used for the simulation are summarized in Supplemental Table 1.

\section{Centriole expansion protocol}

Chlamydomonas reinhardtii centrioles were isolated from the cell-wall-less strain

$567 \mathrm{CW}_{15-{ }^{51}}$ and expanded using the U-ExM protocol ${ }^{31}$. Briefly, isolated centrioles were spun 568 on 12mm Poly-D-Lysine-coated (ThermoFisher, A3890401) coverslips prior to U-ExM. For

569 human cell expansion, cells were initially seeded on $12 \mathrm{~mm}$ coverslips before being fixed

570 with 4\% PFA. Coverslips were then incubated in a solution of $0.7 \%$ formaldehyde and $1 \%$

571 acrylamide in PBS for $4-5$ hours at $37^{\circ} \mathrm{C}$. Next, coverslips were incubated in the monomer

572 solution for 1 minute on ice and then shifted to $37^{\circ} \mathrm{C}$, for 1 hour in a dark and humidified

573 chamber. For one gel, the monomer solution is made of $25 \mu \mathrm{L}$ sodium acrylate (Sigma-

574 Aldrich, 408220, 38\% (wt/wt, diluted with nuclease-free water)), $12.5 \mu \mathrm{L}$ acrylamide (Sigma-

575 Aldrich, A4058, 40\% stock solution), $2.5 \mu \mathrm{L} \mathrm{N}, \mathrm{N}^{\prime}$-methylenbisacrylamide (Sigma-Aldrich,

$576 \mathrm{M} 1533,2 \%$ stock solution), and $5 \mu \mathrm{L}$ 10X PBS, supplemented with $2.5 \mu \mathrm{L}$ TEMED and 2.5

$577 \mu \mathrm{L}$ APS (from 10\% stock solutions). Once polymerized, gels were moved into denaturation

578 buffer (200 mM SDS, $200 \mathrm{mM} \mathrm{NaCl}$ and $50 \mathrm{mM}$ Tris in nuclease-free water, pH 9) for 15

579 minutes at room temperature with gentle shaking and then shifted for 30 minutes to $95^{\circ} \mathrm{C}$ in

580 an $1.5 \mathrm{~mL}$ Eppendorf tube with $1 \mathrm{~mL}$ of fresh denaturation buffer. Then, gels were placed at 
581 room temperature in beakers with $200 \mathrm{~mL}$ of distilled water. Water was exchanged twice

582 (every $30 \mathrm{~min}$ ) and the sample was incubated overnight at room temperature. The following

583 day, water was changed with PBS for $15 \mathrm{~min}$ at room temperature. Gels were then

584 incubated for $3 \mathrm{~h}$ at $37^{\circ} \mathrm{C}$ with gentle shaking in $1 \mathrm{~mL}$ of primary antibody solution (in $3 \%$

585 BSA and $0.05 \%$ Tween20). Samples were then washed three times for 10 min with PBS

586 supplemented by $0.1 \%$ Tween 20 while shaking, followed by incubation with secondary

587 antibodies for $3 \mathrm{~h}$ at $37^{\circ} \mathrm{C}$ in $3 \%$ BSA and $0.05 \%$ Tween20. Finally gels were washed $3 \mathrm{x}$ in

588 T-PBS. For RPE-1 cells, the sample was supplemented in the second wash with Hoechst

$58933258\left(1: 2{ }^{\prime} 000\right)$ dye and place in beakers with $200 \mathrm{~mL}$ of distilled water for final expansion,

590 with again water being exchanged twice for 30'. Before imaging, gels were again expanded

591 overnight in $\mathrm{ddH}_{2} \mathrm{O}$. Primary antibodies used to image centrioles in expanded RPE-1 cells

592 were rabbit anti-polyglutamate chain (polyE), pAb (IN105, 1:250) and mouse monoclonal

593 anti-acetylated tubulin (Merck, T7451, 1:500). Secondary antibodies were goat anti-Rabbit

594 Alexa488 (Thermo-Fisher Scientific, A11034, 1:500) and goat anti-mouse IgG Alexa 568

$595 \mathrm{~F}(\mathrm{ab}) 2$ (Thermo-Fisher Scientific, A11019, 1:500). The following primary antibodies were

596 used for imaging expanded purified Chlamydomonas reinhardtii centrioles: rabbit polyclonal

597 anti-polyglutamate chain (PolyE, IN105,1:500, AG-25B-0030-C050, Adipogen), mouse

598 monoclonal anti- $\alpha$-tubulin (DM1 $\alpha, 1: 500$, T6199, Sigma-Aldrich), mouse monoclonal anti-

599 polyglutamylation modification (GT335,1:200, AG-20B-0020, Adipogen), mouse monoclonal

600 anti-acetyl- $\alpha$-tubulin (Lys40,1:50, 32-2700, Invitrogen, ThermoFisher). Secondary

601 antibodies were goat anti-rabbit Alexa Fluor $488 \operatorname{lgG} H+\mathrm{L}(1: 400, \mathrm{~A} 11008)$, goat anti-mouse

602 Alexa Fluor $488 \operatorname{lgg} \mathrm{H}+\mathrm{L}$ (1:400, A11029), goat anti-rabbit Alexa Fluor $568 \lg \mathrm{H}+\mathrm{L}(1: 400$,

603 A11036), goat anti-mouse Alexa Fluor 568 lgG H+L (1:400, A11004 all from Invitrogen,

604 ThermoFisher).

Particle segmentation, classification and 3D reconstruction of purified centrioles

Particles were segmented using a custom written Matlab script. Briefly, the 607 threshold value was found on the maximum intensity projections of the 3D stacks, before 
608 applying it back to the individual stacks. The regions were then binarized and segmented

609 by connecting neighboring pixels across the 3D stack. The binarized points of interested

610 were then dilated to form the mask which was then applied back to the raw stack. The

611 particles were then cut out from the original stack with their specific mask and a $60 \times 60$ pixel

612 surrounding region. The segmentation was $\sim 50 \%$ successful, mostly because centrioles are

613 often found in pairs, and closely located pairs had to be rejected since they could not be

614 segmented correctly.

615 After particles were segmented and up-sampled (to reach isotropic pixel size), the

616 particles with tubulin signal were aligned to a reference using Dynamo. The reference was

617 built using a reconstructed tubulin signal made from 12 manually selected particles. Once

618 particles were all similarly aligned, a cross-correlation matrix was generated by calculating

619 the similarity between each pairs of particles. The cross-correlation matrix was converted to

620 a distance matrix by subtracting cross-correlation values to 1 . A hierarchical classification

621 was made using the Ward method (hclust function from R). The classification tree was

622 empirically cut into 10 groups. For each group, the average particle was generated for the

623 tubulin signal and for the PolyE signal by applying the transformation parameters obtained

624 during the alignment step. The 10 averages were compared to identify which groups were

625 most promising. The best classes were used to produce the reconstruction results of Figure

626 4. The reconstruction method was based on ref. ${ }^{44}$, and followed the procedure reported in

627 ref. ${ }^{31}$ with a C9 symmetry constraint. The point spread function was experimentally

628 measured by imaging $100 \mathrm{~nm}$ fluorescent beads (TetraSpeck ${ }^{\mathrm{TM}}$ Microspheres, $0.1 \mu \mathrm{m}$,

629 T7279 ThermoFisher). We made two modifications to the reference-free step of the

630 reconstruction algorithm ${ }^{44}$. Firstly, we adopted a multiscale reconstruction approach: we

631 used results obtained with coarsely subsampled data to get a coarse initialization. Thanks

632 to this approach, we were able to perform the reconstruction at the highest resolution in

633 reasonable computation time. Secondly, we decoupled the angular search of the

634 orientations: the first two Euler angles were estimated first, before the third one. This further

635 accelerated the reconstruction and provided sharper results in the case of C9 symmetry. 
Reconstructions were visualized using Chimera, by setting the threshold to fit the

637 average length of the particles. Artefactual signals in the center of the reconstruction that

638 arise due to the imposed 9-fold symmetry were removed during post-processing.

\section{Acknowledgements}

We thank Hari Shroff and Alistair Curd for their help and advice for the construction

641 of the iSIM; Cordelia Berz for her help with multi-FOV imaging; Raoul Kirchner on

642 discussions on the Koehler integrator; Christian Sieben and Lina Carlini for critical reading

643 of the manuscript.

644 This work is supported by the European Research Council (ERC; AdG 835322, 645 CENGIN to P. Gö and ERC; StG 715289 ACCENT to P.Gu), the Swiss National Science 646 Foundation (SNSF) PP00P3_157517 to P. Gu and 182429 to S.M., the MSCA (75200, 647 CARTASSY to N.B.), and the National Centre for Competence in Research (NCCR) 648 Chemical Biology (S.M.).

\section{Author contributions}

D.M., K.M.D., P.Gö., V.H., P.Gu. and S.M. conceived and designed the project.

651 P.Gö., V.H., P.Gu and S.M. supervised the project. D.M., K.M.D. and S.M. designed the 652 multi-focal illumination system. D.M. and K.M.D. developed the simulation platform. D.M. 653 built the microscope, performed all experiments and data analysis. D.G. performed the 654 purified Chlamydomonas reinhardtii centriole sample preparation. D.F. performed single 655 particle averaging and reconstruction. N.B. performed the human RPE-1 centriole sample 656 preparation. M.L.G. performed the particle classification and alignment. K.I. built and 657 performed experiments on the flat-fielding characterization platform. D.M. and S.M. wrote 658 the manuscript with contributions from all authors. 


\section{Competing interests}

660 The authors declare no competing financial interests.

\section{$661 \quad$ References}

662 1. Rust, M. J., Bates, M. \& Zhuang, X. Sub-diffraction-limit imaging by stochastic 663 optical reconstruction microscopy (STORM). Nat. Methods 3, 793-796 (2006).

664 2. Betzig, E. et al. Imaging Intracellular Fluorescent Proteins at Nanometer Resolution. 665 Science (80-. ). 313, 1642-1645 (2006).

666 3. Gustafsson, M. G. L. Surpassing the lateral resolution limit by a factor of two using 667 structured illumination microscopy. J. Microsc. 198, 82-87 (2000).

668 4. Gustafsson, M. G. L. et al. Three-Dimensional Resolution Doubling in Wide-Field 669 Fluorescence Microscopy by Structured Illumination. Biophys. J. 94, 4957-4970 (2008).

670 5. Hell Stefan, W. \& Jan, W. Breaking the diffraction resolution limit by stimulated 671 emission: stimulated-emission-depletion fluorescence microscopy. Opt. Lett 19, 780-2 672 (1994).

673 6. Tanaami, T. et al. High-speed 1-frame/ms scanning confocal microscope with a 674 microlens and Nipkow disks. Appl. Opt. 41, 4704 (2002).

675 7. Bingen, P., Reuss, M., Engelhardt, J. \& Hell, S. W. Parallelized STED fluorescence 676 nanoscopy. Opt. Express 19, 23716 (2011).

677 8. Chmyrov, A. et al. Nanoscopy with more than 100,000 'doughnuts'. Nat. Methods $678 \quad 10,737-740(2013)$

679 9. York, A. G. et al. Instant super-resolution imaging in live cells and embryos via 680 analog image processing. Nat. Methods 10, 1122-1126 (2013).

681 10. Douglass, K. M., Sieben, C., Archetti, A., Lambert, A. \& Manley, S. Super-resolution 682 imaging of multiple cells by optimized flat-field epi-illumination. Nat. Photonics 10, 705-708 683 (2016).

684 11. Archetti, A. et al. Waveguide-PAINT offers an open platform for large field-of-view 
686 12. Chen, F., Tillberg, P. W. \& Boyden, E. S. Expansion microscopy. Science (80-. ). 347, 543-548 (2015).

688 13. Chen, F. et al. Nanoscale imaging of RNA with expansion microscopy. Nat. Methods 13, 679-684 (2016).

690 14. Gao, R. et al. Cortical column and whole-brain imaging with molecular contrast and nanoscale resolution. Science (80-. ). 363, eaau8302 (2019).

692 15. Cahoon, C. K. et al. Superresolution expansion microscopy reveals the three693 dimensional organization of the Drosophila synaptonemal complex. Proc. Natl. Acad. Sci. 694201705623 (2017). doi:10.1073/pnas.1705623114

695 16. Halpern, A. R., Alas, G. C. M., Chozinski, T. J., Paredez, A. R. \& Vaughan, J. C. 696 Hybrid Structured Illumination Expansion Microscopy Reveals Microbial Cytoskeleton 697 Organization. ACS Nano 11, 12677-12686 (2017).

698 17. Gao, M. et al. Expansion Stimulated Emission Depletion Microscopy (ExSTED). 699 ACS Nano 12, 4178-4185 (2018).

700 18. $\mathrm{Xu}, \mathrm{H}$. et al. Molecular organization of mammalian meiotic chromosome axis 701 revealed by expansion STORM microscopy. Proc. Natl. Acad. Sci. 116, 18423-18428 702 (2019).

703 19. Mahecic, D., Testa, I., Griffié, J. \& Manley, S. Strategies for increasing the 704 throughput of super-resolution microscopies. Curr. Opin. Chem. Biol. 51, 84-91 (2019).

705 20. Sacconi, L. et al. Multiphoton multifocal microscopy exploiting a diffractive optical 706 element. Opt. Lett. 28, 1918 (2003).

707 21. Coelho, S. et al. Multifocal multiphoton microscopy with adaptive optical correction. 708 Multiphot. Microsc. Biomed. Sci. XIII 8588, 858817 (2013).

709 22. Cooper, D. J. F. Imaging distal end of multimode fiber. United States Pat. 1, (2014).

710 23. Nielsen, T., Fricke, M., Hellweg, D. \& Andresen, P. High efficiency beam splitter for 711 multifocal multiphoton microscopy. J. Microsc. 201, 368-376 (2001).

712 24. Voelkel, R., Herzig, H. P., Nussbaum, P., Daendliker, R. \& Hugle, W. B. Microlens 
713 array imaging system for photolithography. Soc. Photo-Optical Instrum. Eng. 35, 3323-

$7143330(1996)$.

715 25. Zimmermann, M., Lindlein, N., Voelkel, R. \& Weible, K. J. Microlens laser beam

716 homogenizer: from theory to application. 666302, 666302 (2007).

717 26. Voelkel, R. \& Weible, K. J. Laser beam homogenizing: limitations and constraints.

$718 \quad 71020 \mathrm{~J}(2008)$. doi:10.1117/12.799400

719 27. Schreiber, P., Kudaev, S., Dannberg, P. \& Zeitner, U. D. Homogeneous LED-

720 illumination using microlens arrays. Nonimaging Opt. Effic. Illum. Syst. II 5942, 59420K

721 (2005).

722 28. Model, M. A. \& Blank, J. L. Concentrated dyes as a source of two-dimensional

723 fluorescent field for characterization of a confocal microscope. J. Microsc. 229, 12-16

724 (2008).

725 29. Curd, A. et al. Construction of an instant structured illumination microscope.

726 Methods 88, 37-47 (2015).

727 30. Friedman, J. R. et al. ER tubules mark sites of mitochondrial division. Science (80-.

$728 \quad$ ). 334, 358-362 (2011).

729 31. Gambarotto, D. et al. Imaging cellular ultrastructures using expansion microscopy

730 (U-ExM). Nat. Methods 1 (2018). doi:10.1038/s41592-018-0238-1

731 32. Görlitz, F. et al. easySLM-STED: Stimulated emission depletion microscopy with

732 aberration correction, extended field of view and multiple beam scanning. J. Biophotonics

733 11, 1-11(2018).

734 33. Guichard, P., Chrétien, D., Marco, S. \& Tassin, A. M. Procentriole assembly 735 revealed by cryo-electron tomography. EMBO J. 29, 1565-1572 (2010).

736 34. Banterle, N. \& Gönczy, P. Centriole Biogenesis: From Identifying the Characters to

737 Understanding the Plot. Annu. Rev. Cell Dev. Biol. 33, 23-49 (2017).

738 35. Janke, C. \& Bulinski, J. C. Post-translational regulation of the microtubule

739 cytoskeleton: Mechanisms and functions. Nat. Rev. Mol. Cell Biol. 12, 773-786 (2011).

740 36. Bobinnec, Y. et al. Centriole disassembly in vivo and its effect on centrosome 
741 structure and function in vertebrate cells. J. Cell Biol. 143, 1575-1589 (1998).

742 37. Xu, Z. et al. Microtubules acquire resistance from mechanical breakage through

743 intralumenal acetylation. Science (80-. ). 356, 328-332 (2017).

744 38. Portran, D., Schaedel, L., Xu, Z., Théry, M. \& Nachury, M. V. Tubulin acetylation

745 protects long-lived microtubules against mechanical ageing. Nat. Cell Biol. 19, 391-398

746 (2017).

747 39. Eshun-Wilson, L. et al. Effects of $\alpha$-tubulin acetylation on microtubule structure and

748 stability. Proc. Natl. Acad. Sci. U. S. A. 116, 10366-10371 (2019).

749 40. Wolff, A. et al. Distribution of glutamylated alpha and beta-tubulin in mouse tissues

750 using a specific monoclonal antibody, GT335. Eur. J. Cell Biol. 59, 425-32 (1992).

751 41. Abal, M., Keryer, G. \& Bornens, M. Centrioles resist forces applied on centrosomes 752 during G2/M transition. Biol. Cell 97, 425-434 (2005).

753 42. Hamel, V. et al. Identification of Chlamydomonas Central Core Centriolar Proteins

754 Reveals a Role for Human WDR90 in Ciliogenesis. Curr. Biol. 27, 2486-2498.e6 (2017).

755 43. Bobinnec, Y. et al. Glutamylation of centriole and cytoplasmic tubulin in proliferating 756 non- neuronal cells. Cell Motil. Cytoskeleton 39, 223-232 (1998).

757 44. Fortun, D. et al. Reconstruction from multiple particles for 3D isotropic resolution in 758 fluorescence microscopy. IEEE Trans. Med. Imaging 37, 1235-1246 (2018).

759 45. Sieben, C., Banterle, N., Douglass, K. M., Gönczy, P. \& Manley, S. Multicolor single760 particle reconstruction of protein complexes. Nat. Methods 15, 777-780 (2018).

761 46. Campbell, M. G., Veesler, D., Cheng, A., Potter, C. S. \& Carragher, B. $2.8 \AA$ 762 Resolution Reconstruction of the Thermoplasma Acidophilum 20 S Proteasome Using 763 Cryo-Electron Microscopy. Elife 2015, 1-22 (2015).

764 47. Jiang, J., Pentelute, B. L., Collier, R. J. \& Hong Zhou, Z. Atomic structure of anthrax 765 protective antigen pore elucidates toxin translocation. Nature $\mathbf{5 2 1 , 5 4 5 - 5 4 9 ~ ( 2 0 1 5 ) . ~}$

766 48. Meehl, J. B., Bayless, B. A., Giddings, T. H., Pearson, C. G. \& Winey, M. 767 Tetrahymena Poc1 ensures proper intertriplet microtubule linkages to maintain basal body 768 integrity. Mol. Biol. Cell 27, 2394-2403 (2016). 
769 49. Edelstein, A. D. et al. Advanced methods of microscope control using $\mu$ Manager

770 software. J. Biol. Methods 1, 10 (2014).

771 50. Schindelin, J. et al. Fiji: An open-source platform for biological-image analysis. Nat.

772 Methods 9, 676-682 (2012).

773 51. Klena, N. et al. Isolation and Fluorescence Imaging for Single-particle

774 Reconstruction of Chlamydomonas Centrioles. J. Vis. Exp. (2018).

775 52. Wong, Y. L. et al. Reversible centriole depletion with an inhibitor of Polo-like kinase

776 4. Science $\mathbf{3 4 8 , 1 1 5 5 - 1 1 6 0 ( 2 0 1 5 ) . .}$ 